\title{
Preoperative optimization of the vascular surgery patient
}

This article was published in the following Dove Press journal:

Vascular Health and Risk Management

I July 2015

Number of times this article has been viewed

\author{
Henry T Zhan' \\ Seth T Purcell ${ }^{1,2}$ \\ Ruth L Bush' \\ 'Texas A\&M Health Science Center \\ College of Medicine, Bryan, ${ }^{2}$ Baylor \\ Scott and White, Temple, TX, USA
}

Correspondence: Ruth L Bush Texas A\&M Health Science Center College of Medicine, MS I 3598447 State Highway 47, HPEB 3060, Bryan, TX

77807-3260, USA

Tel +l 9794360223

Fax + I 9794360092

Email rbush@medicine.tamhsc.edu

\begin{abstract}
It is well known that patients who suffer from peripheral (noncardiac) vascular disease often have coexisting atherosclerotic diseases of the heart. This may leave the patients susceptible to major adverse cardiac events, including death, myocardial infarction, unstable angina, and pulmonary edema, during the perioperative time period, in addition to the many other complications they may sustain as they undergo vascular surgery procedures, regardless of whether the procedure is performed as an open or endovascular modality. As these patients are at particularly high risk, up to $16 \%$ in published studies, for postoperative cardiac complications, many proposals and algorithms for perioperative optimization have been suggested and studied in the literature. Moreover, in patients with recent coronary stents, the risk of noncardiac surgery on adverse cardiac events is incremental in the first 6 months following stent implantation. Just as postoperative management of patients is vital to the outcome of a patient, preoperative assessment and optimization may reduce, and possibly completely alleviate, the risks of major postoperative complications, as well as assist in the decision-making process regarding the appropriate surgical and anesthetic management. This review article addresses several tools and therapies that treating physicians may employ to medically optimize a patient before they undergo noncardiac vascular surgery.
\end{abstract}

Keywords: perioperative care, intraoperative care, medical management, risk evaluation/ stratification, medical treatment

\section{Introduction}

The population of patients requiring or electing to undergo a peripheral vascular operation often presents with multiple comorbidities, including chronic cardiac disease. Among the list of complications that may occur with vascular operations, postoperative adverse cardiac events such as myocardial ischemia or infarction are among the most common due to the frequency of coexisting atherosclerotic coronary disease. As these patients are at particularly high risk for postoperative cardiac complications, many proposals and algorithms for perioperative optimization have been suggested and studied in the literature.

The approaches to preoperative optimization have been multifactorial, including many controversial management strategies with conflicting data presented. Several authors have advocated for and against fluid management, pharmacotherapy, and coronary revascularization. Preoperative optimizations of vascular surgery patients will need to include many different strategies and be individualized to each patient; however, a definitive approach is still unclear. These patients have a range of comorbidities, and each patient has varying severity of each comorbidity. In this review, we aim to evaluate 
the current body of knowledge on cardiac optimization of vascular patients before elective vascular operations and make recommendations for the most beneficial approach to these patients.

\section{Assessing cardiac risk}

Prior to any vascular procedure, whether performed in an open or endovascular manner, an assessment of a patient's risk for a cardiac event should be performed. Numerous models designed to assess such risks have been designed. Presently, the most prevalent of such tools is the Revised Cardiac Risk Index (RCRI), also known as the Lee Index. ${ }^{1-3}$ This well-known and well-studied tool stratifies patients into three risk categories (low, intermediate, and high) using six variables. While numerous studies have validated this tool for major noncardiac surgery, its accuracy with respect to noncardiac vascular surgery (NCVS) patients has been called into question due to it being derived from a diverse population undergoing a wide range of surgical procedures with only a small subset undergoing NCVS and matching the typical vascular patient profile. ${ }^{4}$

More recently, the Vascular Study Group of New England (VSGNE) developed the Vascular Study Group of New England Cardiac Risk Index (VSG-CRI) as an "accurate, practical and comprehensive risk prediction model" for patients undergoing NCVS. ${ }^{5}$ The VSG-CRI includes nine variables (age, smoking, insulin-dependent diabetes, coronary artery disease [CAD], congestive heart failure, abnormal cardiac stress test, long-term $\beta$-blocker treatment, chronic obstructive pulmonary disease, and serum creatinine level $\geq 1.8 \mathrm{mg} / \mathrm{dL}$ ). Only four of these variables were included in RCRI (insulin-dependent diabetes, CAD, congestive heart failure, and renal insufficiency). Not only the VSGNE found that RCRI underestimated actual cardiac complications in the vascular population, but also the VSG-CRI accurately predicted the actual risk of cardiac complications across the four procedures studied (carotid endarterectomy, lower extremity bypass, endovascular abdominal aortic aneurysm repair, and open infra-renal abdominal aortic aneurysm repair) for low- and high-risk patients when compared to RCRI. ${ }^{5}$ Thus, it is important to assess the patient's risk for the specific operation they are to undergo, open or endovascular. As many endovascular operations may be performed under local anesthesia only, the risk of a perioperative cardiac event may be lower. However, it is important to risk stratifying the patient, as an endovascular operation may need to be converted to an open procedure or the patient may need an additional or adjunct procedure.
Current American Heart Association/American College of Cardiology (AHA/ACC) recommendations for the assessment of intermediate- and high-risk patients (as defined per RCRI) include performing cardiac exercise test, pharmacologic stress test, and electrocardiograms and assessing the left ventricular function; however, the latter is not as well supported in the literature..$^{6-8}$ Myocardial perfusion imaging using thallium has become a popular method of preoperative cardiac assessment. Unlike exercise stress tests, patients are not limited due to various comorbidities and disabilities. While advantageous in that regard, myocardial scintigraphy can expose the patient to an extraordinarily high amount of radiation, especially if using dual isotope scans. A meta-analysis comparing myocardial scintigraphy and stress echocardiogram showed that stress echocardiograms are twice as accurate as a predictor of postoperative cardiac events. ${ }^{9,10}$ Specifically, thallium imaging was found to have higher rates of false negatives. Therefore, stress echocardiogram, whenever possible, is a superior tool for cardiac risk assessment. Routine cardiac angiography and prophylactic percutaneous cardiac interventions are not recommended except in circumstances in which revascularization is indicated according to existing guidelines. For patients who are deemed to be at low risk, no further cardiac assessment is required.

\section{Fluid management}

Fluid management is an essential component of perioperative surgical management for many surgical specialties, and it is especially important for vascular surgery patients. Many common morbidities from vascular surgery procedures can be attributed to intravascular or extravascular etiologies. These include intravascular loss leading to hemodynamic instability or extravasation of intravascular fluids due to systemic inflammation, both of which can be complicated in patients with pre-existing cardiovascular disease. Historically, pulmonary artery catheters (PACs) were placed to optimize a patient before surgery as well as for hemodynamic monitoring. After studies by Berlauk et al, it was also thought that optimizing certain hemodynamic markers such as cardiac index, pulmonary wedge pressure, and systemic vascular resistance using PAC would improve outcomes of patients undergoing vascular surgery. ${ }^{11}$ However, their results have not been repeated in subsequent studies. Bender et al demonstrated that patients optimized with PAC parameters were not shown to have significantly improved perioperative mortality, decreased complications, or decreased lengths of stay while receiving significantly more fluids compared to those in the control groups. ${ }^{12}$ 
Common postoperative morbidities related to fluid status include compromised cardiac and pulmonary functions, most often related to edema and increased vascular permeability. McArdle et al showed that patients who underwent open abdominal vascular surgery with a restricted perioperative fluid regimen vs standard therapy had fewer complications, reduced morbidity, and shorter length of stay without an increase in hypovolemia-related complications such as renal dysfunction. ${ }^{13}$ Furthermore, these authors reported several cases of acute delirium in standard therapy patients, which was conjectured to be directly caused by cerebral edema secondary to hypervolemia or indirectly caused by cerebral hypoxia secondary to cardiopulmonary dysfunction from hypervolemia. Additionally, a 2012 meta-analysis of studies from as early as 1988 showed that "goal-directed" fluid therapy was more beneficial to patients undergoing major surgeries than "liberal" fluid administration. ${ }^{14}$ In this study of 3,861 patients in 23 randomized controlled trials of various fluid management strategies, not only were more fluid used compared to the respective control arms, but also there were highly different outcomes. More liberal fluid usage was associated with higher rates of pneumonia, pulmonary edema, and corresponding longer hospital stays. ${ }^{14}$

Conversely, a broad meta-analysis of 14 randomized control trials from 2007 to 2013 showed that patients treated with postoperative goal-directed fluid therapy based on dynamic parameters experienced significantly less complications as well as shorter Intensive Care Unit stays, although overall length of hospital stay did not decrease. ${ }^{15}$ The authors highlight the use of dynamic parameters such as stroke volume variation to optimize hemodynamic status. However, promising, additional studies are required on the use of dynamic parameters as well as the values of dynamic parameters, especially in vascular surgery.

\section{Pharmacotherapy Beta-blocker usage}

Beta-blockers are one of the most commonly used medications, and their use has been widely studied in vascular surgery patients, a population who may be especially prone to cardiac complications. Perioperative beta-blocking agents have been found to reduce adverse cardiac events as well as deaths. The most notable exception to this was the POISE trial, which showed that while extended-release metoprolol reduced the risks of myocardial infarction, cardiac revascularization, and atrial fibrillation, it also increased the incidence of bradycardia, hypotension, stroke, and death. ${ }^{16}$ However, this large randomized controlled trial performed in 190 hospitals in 23 countries utilized a generalized regimen of metoprolol as well as higher than normal dosages, which are thought to have contributed to many of the reported negative effects. Aside from the dosage of beta-blocker, the preoperative timing of administering beta-blockers has also been shown to play a role in postoperative complications. In the POISE trial, metoprolol was administered 2-4 hours before the beginning of the procedure. Ellenberger et al further studied the timing of preoperative beta-blockers using propensity score matching to create 301 patient pairs who were well balanced for major comorbidities, concomitant drug use, and type of surgery. ${ }^{17}$ These authors found that in patients undergoing elective noncardiac surgery, acute betablockade (started within 2 days after surgery) was associated with significantly higher incidence of postoperative adverse cardiac events than chronic beta-blockade.

We recognize that the data concerning beta-blockade has been controversial, with studies showing the shortterm benefits but others demonstrating a possible increased postoperative risk of mortality with chronic usage. $5,16,17$ The VSGNE worked to increase the percentage of patients given beta-blocking agents before elective vascular surgery. ${ }^{18}$ However, in the VSG-CRI, long-term beta-blockade was one of the nine factors contributing for an increased postoperative risk of major adverse cardiac events (MACEs). ${ }^{5}$ Lindenauer et al found in a large retrospective study published in 2005 that "perioperative beta-blocker therapy was associated with a reduced risk of in-hospital death among high-risk, but not low-risk, patients undergoing major noncardiac surgery". ${ }^{19}$ Thus, it appears from the literature that beta-blockade should be used in specific high-risk individuals. The literature supports judicious perioperative beta-blocker administration in those deemed at high or highest risk for an elective vascular procedure.

\section{Pharmacotherapy - antiplatelet therapy}

There are many indications for antiplatelet therapy, and consequently, antiplatelet therapy is common among vascular surgery patients. For patients about to undergo vascular surgery, a careful decision needs to be made regarding the patients' anticoagulation status. Just as vascular patients require anticoagulation after peripheral vascular interventions, it is important to ascertain if the patient has had coronary stents implanted, and if so, when. The most recent $\mathrm{AHA} / \mathrm{ACC}$ guidelines recommend, if possible, to delay surgery until after 4-6 weeks after stent implantation, the reason being that risks of coronary stent thrombosis, and consequently, MACEs are the highest during that time. ${ }^{20}$ If 
surgery is urgent, dual antiplatelet therapy should not be discontinued unless the risk of bleeding outweighs the benefit of stent thrombosis. ${ }^{20,21}$ For patients with coronary stents after this period who requires elective noncardiac surgery, recent studies have shown to be in favor of continuing aspirin. The POISE-2 trial demonstrated that while rates of major intraoperative bleeding is significantly higher in the aspirin group compared to the placebo group, mortality rate and rate of cardiac events were insignificant between the two groups. ${ }^{22}$ Calderaro et al had similar conclusions as the POISE-2 trial, and they further suggest that aspirin responsiveness is possibly related to cardiovascular events. ${ }^{23}$ In addition to aspirin, thienopyridine-class antiplatelet agents are also employed frequently in vascular patients. ${ }^{24}$ Saadeh and Sfeir found that the continuation of clopidogrel was not associated with increased bleeding complications in various vascular procedures, although the standard practice is to traditionally discontinue this particular agent. ${ }^{25}$

In addition to aspirin and thienopyridine-class antiplatelet agents, novel anticoagulation agents are being used more. While these new agents have their own advantages, disadvantages such as lack of a reversing agent are important factors to consider in the evaluation of preoperative patients. Currently, there have been no published studies involving these agents and vascular patients. However, in other noncardiac surgery studies, patients are stratified based on the risk of bleeding, the risk of thrombosis, and the procedure involved, and high-risk patients are bridged over to low-molecular-weight heparin before surgery. Consultation to cardiology and open communication between the surgeon and the patient are also important in making medication changes before surgery.

\section{Preoperative cardiac revascularization}

Generally, patients undergoing elective vascular surgery often suffer from coexisting CAD, as evidenced by the high rates found in preoperative screening and the incidence of perioperative adverse cardiac events. In addition to assessing the patients for risk of cardiac events, cardiac-specific issues (such as the need for cardiac revascularization) should be addressed before elective vascular surgery to mitigate these risks. Initial practice had been to screen for and, if necessary, undergo cardiac revascularization before proceeding to treat the peripheral vascular disease. While the newest American Heart Association (AHA) guidelines do not recommend routine invasive catheterizations and revascularizations, ${ }^{7}$ there is still controversy surrounding preoperative assessment of a vascular surgery patient. McFalls et al in the CARP trial demonstrated that revascularization before elective vascular surgery does not significantly improve long-term outcomes. ${ }^{26}$ Patients with CAD that was clinically significant but stable, who underwent routine revascularization, did not experience an improvement in either overall mortality or other outcomes such as postoperative myocardial infarction, stroke, limb loss, or renal failure. In a different study, in patients with medium-to-high risks, Monaco et al found that patients who underwent routine coronary angiography and necessary subsequent revascularization have better postoperative outcomes compared to those who were first screened by noninvasive stress imaging tests. ${ }^{27}$ Thus, there continues to be controversy surrounding the role of cardiac revascularization of patients before vascular surgery. Nonetheless, it is crucial to assess an individual patient's status relative to cardiac disease and the stability of their cardiac disease, and proceed accordingly.

\section{Remote ischemic preconditioning}

Ischemic preconditioning is a phenomenon that was first described in 1986 in which ischemic myocardial tissue is protected by preceding, sublethal ischemic events with intermittent reperfusion. ${ }^{28,29}$ This concept has been applied to patients undergoing cardiac surgery with positive results. ${ }^{30,31}$ Remote ischemic preconditioning (RIPC) works at end organs under the same principle to offer similar protection to other organ systems. ${ }^{32-34}$ For patients requiring cardiac revascularization, the Cardiac Remote Ischemic Preconditioning in Coronary Stenting study showed a significant decrease in the incidence of MACEs. ${ }^{31}$ In peripheral vascular patients, RIPC before elective open abdominal aortic aneurysm repair induced by sequential cross-clamping of both common iliac arteries for 10 minutes with an equal period of reperfusion before cross-clamping the aorta reduced myocardial injury by $27 \%$ and myocardial infarction by $22 \% .{ }^{35}$ An ongoing study (CRIPES trial; clinical trials.gov identifier NCT01403337) aims to study the effect of RIPC using upper arm compression for 24 hours before elective major vascular surgery. Outcomes from that study are currently pending.

\section{Renal insufficiency}

Similar to how vascular patients tend to have cardiac comorbidities, renal disease from a variety of pathologies is a common comorbidity as well. For these patients, the presence or the absence of anemia, thrombocytopenia, electrolyte imbalances, and acid-base abnormalities should be determined and addressed before surgery. ${ }^{36}$ For patients with endstage renal disease approaching the need for hemodialysis, Gajdos et al found that these patients, notably 65 years or 
older, have especially poor postoperative prognosis and argue for a higher threshold of major vascular surgery for this patient population. ${ }^{37}$ Additionally, a baseline renal function should be determined as renal insufficiency that can contribute to postoperative complications. This is especially important in endovascular procedures, in which chronic kidney disease patients are especially susceptible to contrastinduced nephropathy. For patients whose kidney functions could not tolerate typical contrast, gadolinium-based contrast is offered as an alternative. Although rare, surgeons should be aware of the condition known as nephrogenic systemic fibrosis or nephrogenic fibrosing dermopathy, in which patients develop systemic fibrosis, brought on by the use of gadolinium-based contrast. Lastly, renal artery stenting is not recommended for patients with known renal artery stenosis. The 2014 CORAL trial showed no significant improvement in adverse cardiovascular and renal events in patients who underwent stenting and medical management compared to medical management alone. ${ }^{38}$

\section{Carotid disease}

The practice of routine screening for significant carotid stenosis has been addressed in the literature often with controversial recommendations. The expectation of routine screening is to ultimately identify patients who may benefit from prophylactic carotid interventions to reduce the risk of a perioperative neurologic event with an elective vascular procedure. The majority of the literature has focused on the need for carotid procedures before or concomitantly with coronary artery bypass grafting. ${ }^{39-41}$ The decision is clear for patients who have a significant symptomatic carotid stenosis; these patients require urgent intervention. However, in 2015, the management of an asymptomatic carotid stenosis is the subject of much controversy. ${ }^{42-45}$ Many studies now advocate for modern best medical management in the patients with asymptomatic carotid disease. As stated by Naylor, "a small cohort of 'high-risk for stroke' patients will undoubtedly benefit from intervention and our goal must be to identify and treat these individuals, rather than continuing with a policy of mass intervention that benefits very few patients in the long term". 46

\section{Conclusion}

There has been tremendous progress in reducing the morbidity and mortality that typically surrounds vascular surgery procedures, and preoperative assessment and optimization have played a significant role therein. Additionally, preoperative optimization may require consultants in other specialties. A meticulous physician must recognize the need for a multidisciplinary approach when necessary. Risk scoring systems, while cumbersome, should direct optimization by determining whether patient is at low, medium, or high risk for perioperative adverse cardiac events. By having more specific data and risk stratification systems, such as the predictability of the VSG-CRI, ${ }^{5}$ surgeons and anesthesiologists can have improved clinical decision making. Published guidelines have excellent information and guidance, but one first needs to assess the patient to apply the correct treatment algorithm. ${ }^{7}$ Just as outstanding technical surgical skills are vital to patient outcomes, careful perioperative assessment and clinical management are equally important tools to ensuring excellent clinical outcomes and low complication profiles.

\section{Disclosure}

The authors report no conflicts of interest in this work.

\section{References}

1. Archan S, Roscher CR, Fairman RM, Fleisher LA. Revised cardiac risk index (Lee) and perioperative cardiac events as predictors of long-term mortality in patients undergoing endovascular abdominal aortic aneurysm repair. J Cardiothorac Vasc Anesth. 2010;24(1):84-90.

2. Ford MK, Beattie WS, Wijeysundera DN. Systematic review: prediction of perioperative cardiac complications and mortality by the revised cardiac risk index. Ann Intern Med. 2010;152(1):26-35.

3. Galyfos G, Filis K. Is the revised cardiac risk index the right risk index for vascular surgery patients? Crit Care Med. 2014;42(9):e631-e632.

4. Payne CJ, Bryce GJ, Gibson SC, Kingsmore DB. The revised cardiac risk index performs poorly in patients undergoing major vascular surgery: a prospective observational study. Eur J Anaesthesiol. 2013;30(11):713-715.

5. Bertges DJ, Goodney PP, Zhao Y, et al; Vascular Study Group of New England. The Vascular Study Group of New England Cardiac Risk Index (VSG-CRI) predicts cardiac complications more accurately than the revised cardiac risk index in vascular surgery patients. J Vasc Surg. 2010;52(3):e1-e83.

6. Fleisher LA, Fleischmann KE, Auerbach AD, et al; American College of Cardiology; American Heart Association. 2014 ACC/AHA guideline on perioperative cardiovascular evaluation and management of patients undergoing noncardiac surgery: a report of the American College of Cardiology/American Heart Association Task Force on Practice Guidelines. J Am Coll Cardiol. 2014;64(22):e77-e137.

7. Fleisher LA, Fleischmann KE, Auerbach AD, et al. 2014 ACC/AHA guideline on perioperative cardiovascular evaluation and management of patients undergoing noncardiac surgery: executive summary: a report of the American College of Cardiology/American Heart Association Task Force on Practice Guidelines. Circulation. 2014;130(24):2215-2245.

8. Wijeysundera DN, Duncan D, Nkonde-Price C, et al; ACC/AHA Task Force Members. Perioperative beta blockade in noncardiac surgery: a systematic review for the $2014 \mathrm{ACC} / \mathrm{AHA}$ guideline on perioperative cardiovascular evaluation and management of patients undergoing noncardiac surgery: a report of the American College of Cardiology/ American Heart Association Task Force on Practice Guidelines. Circulation. 2014;130(24):2246-2264.

9. Beattie WS, Abdelnaem E, Wijeysundera DN, Buckley DN A meta-analytic comparison of preoperative stress echocardiography and nuclear scintigraphy imaging. Anesth Analg. 2006;102(1):8-16. 
10. Einstein AJ, Moser KW, Thompson RC, Cerqueira MD, Henzlova MJ. Radiation dose to patients from cardiac diagnostic imaging. Circulation. 2007;116(11):1290-1305.

11. Berlauk JF, Abrams JH, Gilmour IJ, O'Connor SR, Knighton DR, Cerra FB. Preoperative optimization of cardiovascular hemodynamics improves outcome in peripheral vascular surgery. A prospective, randomized clinical trial. Ann Surg. 1991;214(3):289-297; discussion 98-99.

12. Bender JS, Smith-Meek MA, Jones CE. Routine pulmonary artery catheterization does not reduce morbidity and mortality of elective vascular surgery: results of a prospective, randomized trial. Ann Surg. 1997;226(3):229-236; discussion 36-37.

13. McArdle GT, McAuley DF, McKinley A, Blair P, Hoper M, Harkin DW. Preliminary results of a prospective randomized trial of restrictive versus standard fluid regime in elective open abdominal aortic aneurysm repair. Ann Surg. 2009;250(1):28-34.

14. Corcoran T, Rhodes JE, Clarke S, Myles PS, Ho KM. Perioperative fluid management strategies in major surgery: a stratified meta-analysis. Anesth Analg. 2012;114(3):640-651.

15. Benes J, Giglio M, Brienza N, Michard F. The effects of goal-directed fluid therapy based on dynamic parameters on post-surgical outcome: a metaanalysis of randomized controlled trials. Crit Care. 2014;18(5):584.

16. POISE Study Group; Devereaux PJ, Yang H, et al. Effects of extendedrelease metoprolol succinate in patients undergoing non-cardiac surgery (POISE trial): a randomised controlled trial. Lancet. 2008; 371(9627):1839-1847.

17. Ellenberger C, Tait G, Beattie WS. Chronic beta blockade is associated with a better outcome after elective noncardiac surgery than acute beta blockade: a single-center propensity-matched cohort study. Anesthesiology. 2011;114(4):817-823.

18. Goodney PP, Eldrup-Jorgensen J, Nolan BW, et al; Vascular Study Group of New England. A regional quality improvement effort to increase beta blocker administration before vascular surgery. $J$ Vasc Surg. 2011;53(5):1316-1328. e1; discussion 27-28.

19. Lindenauer PK, Pekow P, Wang K, Mamidi DK, Gutierrez B, Benjamin EM. Perioperative beta-blocker therapy and mortality after major noncardiac surgery. $N$ Engl J Med. 2005;353(4):349-361.

20. Fleisher LA, Fleischmann KE, Auerbach AD, et al. 2014 ACC/AHA guideline on perioperative cardiovascular evaluation and management of patients undergoing noncardiac surgery: executive summary: a report of the American College of Cardiology/American Heart Association Task Force on Practice Guidelines. Developed in collaboration with the American College of Surgeons, American Society of Anesthesiologists, American Society of Echocardiography, American Society of Nuclear Cardiology, Heart Rhythm Society, Society for Cardiovascular Angiography and Interventions, Society of Cardiovascular Anesthesiologists, and Society of Vascular Medicine Endorsed by the Society of Hospital Medicine. J Nucl Cardiol. 2015;22(1):162-215.

21. Reddy PR, Vaitkus PT. Risks of noncardiac surgery after coronary stenting. Am J Cardiol. 2005;95(6):755-757.

22. Devereaux PJ, Mrkobrada M, Sessler DI, et al; POISE-2 Investigators. Aspirin in patients undergoing noncardiac surgery. $N$ Engl $J$ Med. 2014;370(16):1494-1503.

23. Calderaro D, Pastana AF, Flores da Rocha TR, et al. Aspirin responsiveness safely lowers perioperative cardiovascular risk. J Vasc Surg. 2013;58(6):1593-1599.

24. Gandhi NK, Abdel-Karim AR, Banerjee S, Brilakis ES. Frequency and risk of noncardiac surgery after drug-eluting stent implantation. Catheter Cardiovasc Interv. 2011;77(7):972-976.

25. Saadeh C, Sfeir J. Discontinuation of preoperative clopidogrel is unnecessary in peripheral arterial surgery. J Vasc Surg. 2013;58(6): 1586-1592.

26. McFalls EO, Ward HB, Moritz TE, et al. Coronary-artery revascularization before elective major vascular surgery. $N$ Engl J Med. 2004; 351(27):2795-2804.

27. Monaco M, Stassano P, Di Tommaso L, et al. Systematic strategy of prophylactic coronary angiography improves long-term outcome after major vascular surgery in medium- to high-risk patients: a prospective, randomized study. J Am Coll Cardiol. 2009;54(11):989-996.
28. Ali ZA, Callaghan CJ, Lim E, et al. Remote ischemic preconditioning reduces myocardial and renal injury after elective abdominal aortic aneurysm repair: a randomized controlled trial. Circulation. 2007; 116(11 Suppl):I98-I105.

29. Murry CE, Jennings RB, Reimer KA. Preconditioning with ischemia: a delay of lethal cell injury in ischemic myocardium. Circulation. 1986; 74(5):1124-1136.

30. Hudetz JA, Patterson KM, Iqbal Z, Gandhi SD, Pagel PS. Remote ischemic preconditioning prevents deterioration of short-term postoperative cognitive function after cardiac surgery using cardiopulmonary bypass: results of a pilot investigation. J Cardiothorac Vasc Anesth. 2014;29(2):382-388.

31. Hoole SP, Heck PM, Sharples L, et al. Cardiac remote ischemic preconditioning in coronary stenting (CRISP stent) study: a prospective, randomized control trial. Circulation. 2009;119(6):820-827.

32. Ferko M, Kancirová I, Jašová M, et al. Remote ischemic preconditioning of the heart: protective responses in functional and biophysical properties of cardiac mitochondria. Physiol Res. 2015;63(Suppl 4):S469-S478.

33. McCrindle BW, Clarizia NA, Khaikin S, et al. Remote ischemic preconditioning in children undergoing cardiac surgery with cardiopulmonary bypass: a single-center double-blinded randomized trial. J Am Heart Assoc. 2014;3(4):e000964.

34. Zimmerman RF, Ezeanuna PU, Kane JC, et al. Ischemic preconditioning at a remote site prevents acute kidney injury in patients following cardiac surgery. Kidney Int. 2011;80(8):861-867.

35. Li L, Li G, Yu C, Li Y. The role of remote ischemic preconditioning on postoperative kidney injury in patients undergoing cardiac and vascular interventions: a meta-analysis. J Cardiothorac Surg. 2013;8:43.

36. Singh S, Maldonado Y, Taylor MA. Optimal perioperative medical management of the vascular surgery patient. Anesthesiol Clin. 2014; 32(3):615-637.

37. Gajdos C, Hawn MT, Kile D, et al. The risk of major elective vascular surgical procedures in patients with end-stage renal disease. Ann Surg. 2013;257(4):766-773.

38. Cooper CJ, Murphy TP, Cutlip DE, et al; CORAL Investigators. Stenting and medical therapy for atherosclerotic renal-artery stenosis. $N$ Engl $J$ Med. 2014;370(1):13-22.

39. Naylor AR. How robust is the evidence supporting prophylactic carotid endarterectomy in patients undergoing coronary bypass? Acta Chir Belg. 2004;104(6):626-629.

40. Peiper C, Nowack J, Ktenidis K, Hopstein S, Keresztury G, Horsch S. Prophylactic urgent revascularization of the internal carotid artery in the symptomatic patient. Vasa. 2001;30(4):247-251.

41. Hines GL, Scott WC, Schubach SL, Kofsky E, Wehbe U, Cabasino E. Prophylactic carotid endarterectomy in patients with high-grade carotid stenosis undergoing coronary bypass: does it decrease the incidence of perioperative stroke? Ann Vasc Surg. 1998;12(1):23-27.

42. Ballotta E, Renon L, Da Giau G, Barbon B, De Rossi A, Baracchini C. Prospective randomized study on asymptomatic severe carotid stenosis and perioperative stroke risk in patients undergoing major vascular surgery: prophylactic or deferred carotid endarterectomy? Ann Vasc Surg. 2005;19(6):876-881.

43. Naylor AR. Why is the management of asymptomatic carotid disease so controversial? Surgeon. 2015;13(1):34-43.

44. Naylor AR. What is the current status of angioplasty vs endarterectomy in patients with asymptomatic carotid artery disease? $J$ Cardiovasc Surg. 2007;48(2):161-180.

45. Stansby G, Macdonald S, Allison R, et al. Asymptomatic carotid disease and cardiac surgery consensus. Angiology. 2011;62(6):457-460.

46. Naylor AR. Time to rethink management strategies in asymptomatic carotid artery disease. Nat Rev Cardiol. 2012;9(2):116-124. 
Vascular Health and Risk Management

Dovepress

\section{Publish your work in this journal}

Vascular Health and Risk Management is an international, peerreviewed journal of therapeutics and risk management, focusing on concise rapid reporting of clinical studies on the processes involved in the maintenance of vascular health; the monitoring, prevention and treatment of vascular disease and its sequelae; and the involvement of metabolic disorders, particularly diabetes. This journal is indexed on PubMed Central and MedLine. The manuscript management system is completely online and includes a very quick and fair peer-review system, which is all easy to use. Visit http://www.dovepress.com/ testimonials.php to read real quotes from published authors.

Submit your manuscript here: http://www.dovepress.com/vascular-health-and-risk-management-journal 